\title{
Pringle maneuver during open partial nephrectomy for renal tumors
}

\author{
Adrian HASEGAN ${ }^{1}$, Dan BRATU ${ }^{2}$, Valentin PIRVUT ${ }^{1}$, lonela MIHAI ${ }^{1}$, Nicolae GRIGORE ${ }^{1}$ \\ ${ }^{1}$ Department of Urology, Faculty of Medicine, "Lucian Blaga“ University of Sibiu, Romania \\ ${ }^{2}$ Department of General Surgery, Faculty of Medicine, "Lucian Blaga" University of Sibiu, Romania
}

\begin{abstract}
Introduction. Sparing kidney tissue is important because studies show that patients who have their entire kidney removed are more likely to suffer from chronic kidney disease after surgery than patients who received a kidney-sparing partial nephrectomy. The aim of study is to show the effectives of Pringle maneuver for clamping renal pedicle in order to minimize warm ischemia during open partial nephrectomy.

Material and method. We retrospectively analyzed 34 patients diagnosed with cT1 renal tumors between 2012 and 2016 and underwent open partial nephrectomy in Sibiu Urology Department. During the surgery we perform clamping of renal pedicle with Satinsky clamp or with the Pringle maneuver. We analyzed the flowing parameters: operation time, blood loss, clamping time and serum creatinine before and after the surgery.

Results. The mean age of patient with partial nephrectomy was $58.7 \pm 14.7$ years, with $56.7 \pm 12.4$ years for Satinsky group and $60.5 \pm 11.6$ for Pringle group. The average duration for partial nephrectomy was 94.8 \pm 19.4 minutes. Average blood loss for all surgeries was $220 \pm 30 \mathrm{ml}$.

Conclusion. Pringle maneuver is a safe and effective method with low intra and postoperative complications during open partial nephrectomy, having a good control of the renal pedicle with reduced ischemia. Is a feasible method of choice in renal tumor pathology on a single congenital or surgical kidney, mediorenal tumors or near the sinus and multifocal tumors.
\end{abstract}

Keywords: renal cell carcinoma, Pringle maneuver, partial nephrectomy, warm ischemia

\section{INTRODUCTION}

Renal cell carcinoma (RCC) is the most common solid lesion within the kidney and accounts for approximately $90 \%$ of all kidney malignancies (1).

Due to the increased detection of tumours by imaging techniques such as ultrasound and computed tomography (CT), the number of incidentally diagnosed RCCs has increased. These tumours are more often smaller and of lower stage. Current evidence studies found no significant difference in survival between open partial and open radical nephrectomy for small tumours $(\leq 4 \mathrm{~cm})$. For larger tumours $(>4 \mathrm{~cm})$, survival outcomes appeared similar between partial and radical nephrectomy. In all studies where renal function was reported (including one RCT), partial nephrectomy was associated with better preservation of renal function compared with radical nephrectomy. Sparing kidney tissue is important because studies show that patients who have their entire kidney removed are more likely to suffer from chronic kidney disease (CKD) after surgery than patients who received a kidney-sparing partial nephrectomy (2).

The aim of study is to show the effectives of Pringle maneuver for clamping renal pedicle in order to minimize warm ischemia during partial nephrectomy. 
Standard indications for kidney sparing partial nephrectomy: absolute (congenital, surgical and functional solitary kidney), relative (contralateral kidney affected by a pathology that may lead to renal failure in the near future), elective (unilateral kidney tumor with normal contralateral kidney) (3).

\section{MATERIAL AND METHOD}

Between 2012-2016 had partial nephrectomy in our department for renal tumors a total of 42 patients with stage T1 disease.

During the surgery we perform clamping of renal pedicle with Satinsky clamp (20 p) or with the Pringle maneuver (22 p). We analized the flowing parameters: operation time, blood loss, clamping time and serum creatinine before and after the surgery.

Diagnosis and staging protocol involves: clinical examination, ultrasonography, renal function tests, intravenous urography, abdominopelvic computer tomography scan.

The Preoperative Aspects and Dimensions Used for an Anatomical (P.A.D.U.A.) scoring and the R.E.N.A.L. (radius, exophytic/endophytic properties, nearness of tumor to the collecting system or sinus in millimeters, anterior/posterior location relative to polar lines) scoring have been developed in the effort to predict the complications in patients undergoing partial nephrectomy, with an important role on surgical decision-making $(4,5)$.

Partial nephrectomy technique involves:

After induction of general anesthesia with endotracheal intubation anesthesia, the patient is re-positioned in the lateral decubitus position with the thorax $35^{\circ}-40^{\circ}$ rotated. Partial nephrectomy was achieved by retroperitoneal approach, with the 12 th rib resection. After the dissection of the anterior face of the psoas muscle to the level of the large vessels, the ureter and the gonadal vessels were identified. The ascending dissection at this level leads to the identification of the kidney pedicle. After dissection of the renal pedicle was practiced upper pole dissection followed by the identification of renal tumor.

Tumor excision was carried out with the fat that surrounds after blocking the blood flow with the vascular clamp Satinsky or Pringle maneuver (Fig. 1, 2).

After tumor resection, the collector system was sutured, if it was opened, with resorbable thread 4.0. The arteries and veins opened during excision were sutured separately to avoid arterio-venous fistulas or pseudoaneurysms. Renal parenchyma was sutured with interrupted Vicryl 3.0 (Fig. 3). Completion of haemostasis after renal pedicle restoration was improved with haemostatic agents (Gelaspon) (Fig. 4, 5, 6).

The statistical analysis of this paper was done by using chi-square test, the Fischer exact test and the Mann-Whitney $U$ test for parametric variables. A p value $<0.05$ was considered statistically significant.

\section{RESULTS}

The mean age of patient with partial nephrectomy was $58.7 \pm 14.7$ years, with $56.7 \pm 12.4$ years for Satinsky group and $60.5 \pm 11.6$ for Pringle group. Regardind the tumor location the results are similar between the two groups. Patients preoperative characteristics about tumor size, PADUA score and nephrometry score are shown in Table 1.

The average duration for partial nephrectomy was $94.8 \pm 19.4$ minutes, with an average time of $94.3 \pm 18.9$ minutes for surgeries were it was used Satinsky clamp and $95.3 \pm 19.8$ minutes
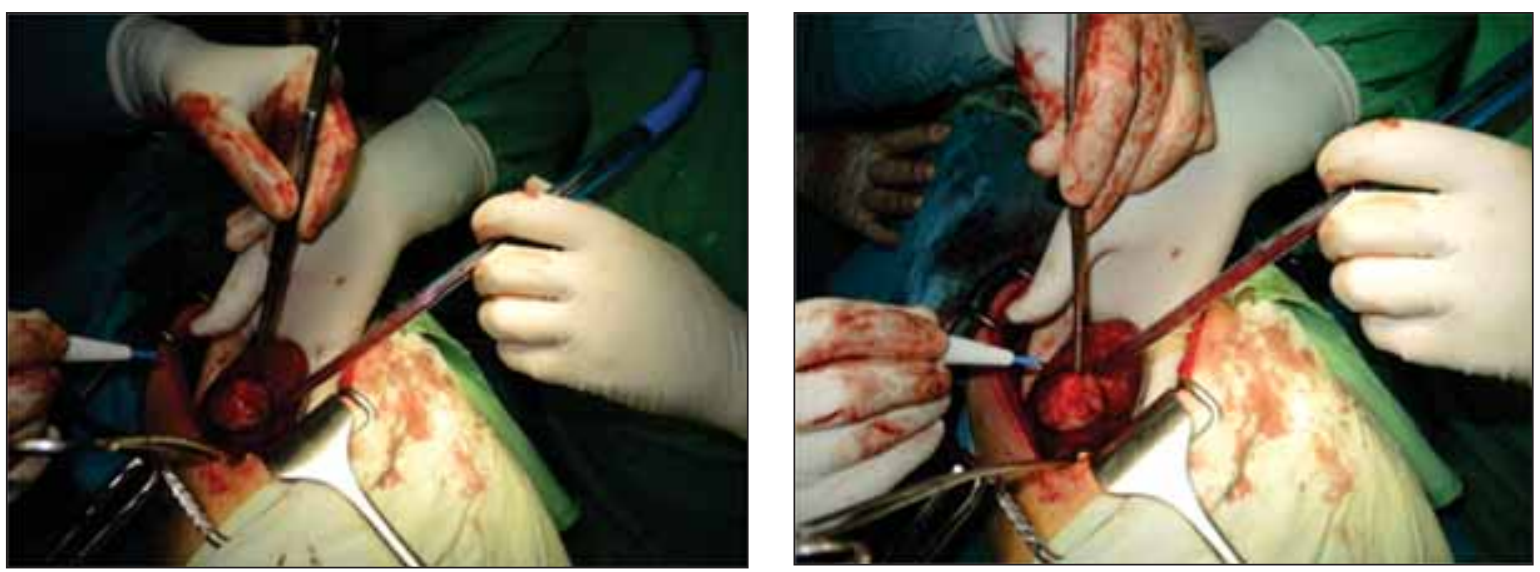

FIGURE 1, 2. Tumor excision after blocking the blood flow with Pringle maneuver 

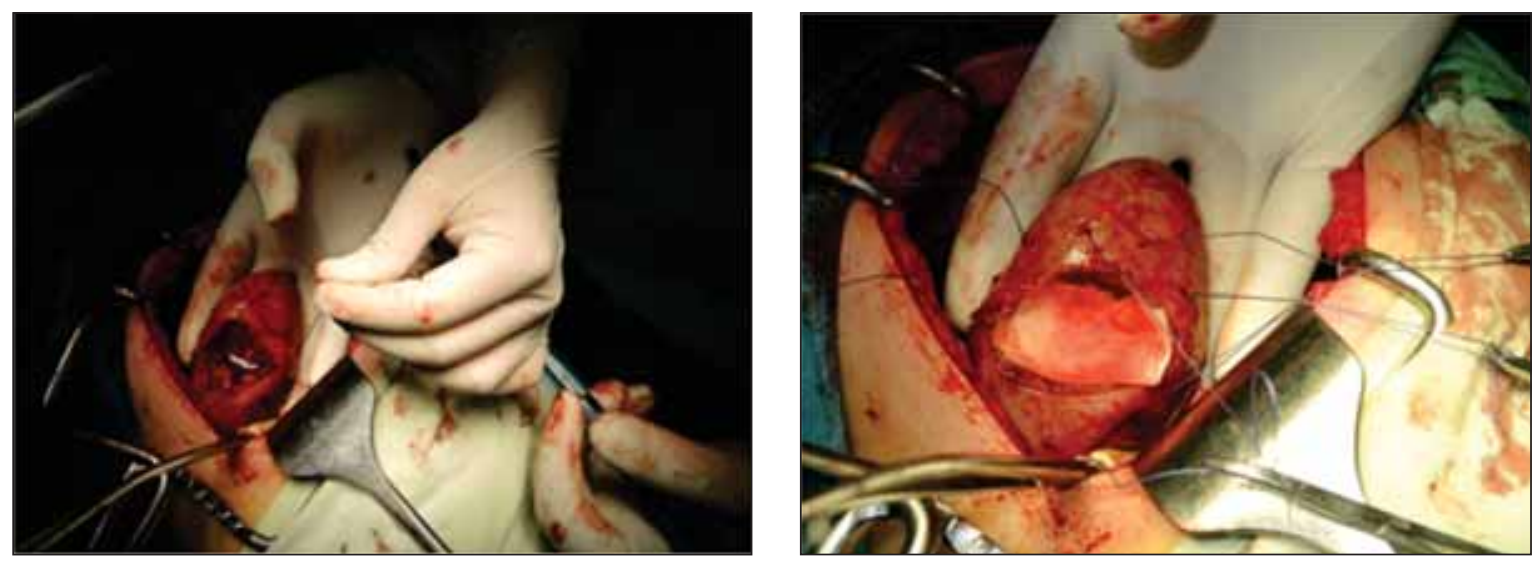

FIGURE 3, 4. Renal parenchyma suture with interrupted Vicryl, and completion of haemostasis with Gelaspone

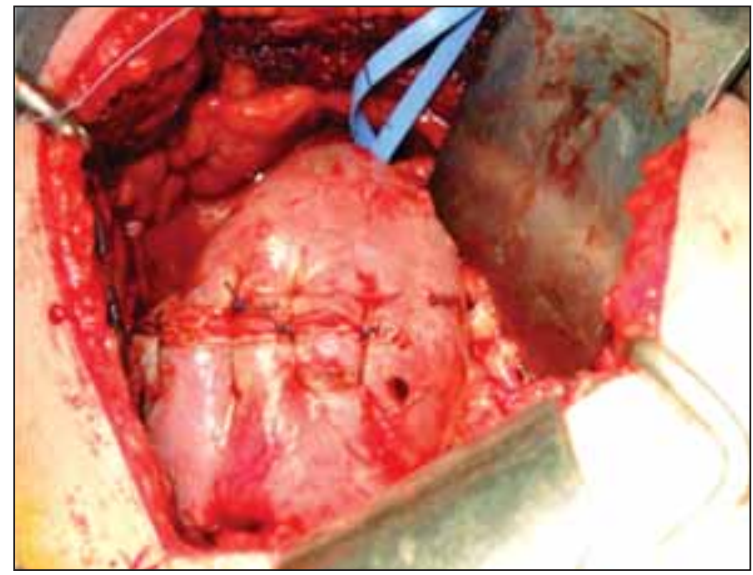

FIGURE 5. The final suture of the renal parenchyma

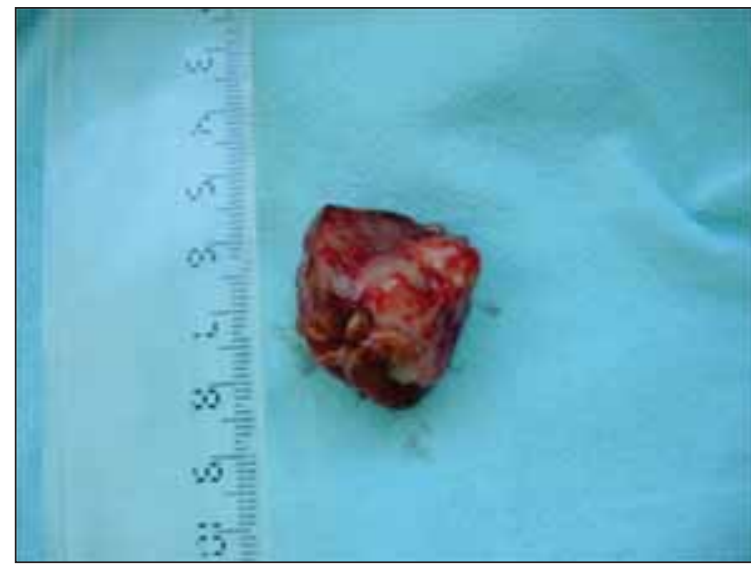

FIGURE 6. Postoperative specimen

TABLE 1. Characteristisc regarding the tumor location

\begin{tabular}{|c|c|c|c|}
\hline Patiens & Satinsky group & Pringle group & p \\
\hline Mean age & $56.7 \pm 12.4(32-74)$ & $60.5 \pm 11.6(29-75)$ & ns \\
\hline \multicolumn{4}{|l|}{ Tumor side } \\
\hline \begin{tabular}{l|l} 
Right \\
\end{tabular} & $8 p(40 \%)$ & $7 \mathrm{p} \mathrm{(31.81 \% )}$ & ns \\
\hline Left & $12 \mathrm{p}(60 \%)$ & $15 \mathrm{p} \mathrm{(68.19 \% )}$ & ns \\
\hline Bilateral & $2 p(10 \%)$ & $3 p(13.64 \%)$ & ns \\
\hline Unique kidney & $2 p(10 \%)$ & $4 \mathrm{p}(18.19 \%)$ & ns \\
\hline \multicolumn{4}{|l|}{ Tumor location } \\
\hline \begin{tabular}{l|l} 
& Upper pole \\
\end{tabular} & $4 p(20 \%)$ & $5 p(22.73 \%)$ & ns \\
\hline Mesorenal & $6 p(30 \%)$ & $7 \mathrm{p} \mathrm{(31.81 \% )}$ & ns \\
\hline Lower pole & $7 p(35 \%)$ & $6 p(27.29 \%)$ & ns \\
\hline Tumor multifocality (2-4) & $3 p(15 \%)$ & $4 \mathrm{p}(18.19 \%)$ & ns \\
\hline Tumor size & $3.9 \pm 1.9(1.5-6.2)$ & $3.4 \pm 1.42(2.4-7.3)$ & ns \\
\hline \multicolumn{4}{|l|}{ P.A.D.U.A score } \\
\hline \begin{tabular}{|l|l|} 
& Low (6-7) \\
\end{tabular} & $9 p(45 \%)$ & $8 p(36.37 \%)$ & ns \\
\hline Medium (8-9) & $7 p(35 \%)$ & $8 p(36.37 \%)$ & ns \\
\hline High (10-14) & $4 p(20 \%)$ & $6 \mathrm{p}(27.29 \%)$ & ns \\
\hline \multicolumn{4}{|l|}{ R.E.N.A.L nephrometry } \\
\hline \begin{tabular}{l|l} 
& Low (4-6) \\
\end{tabular} & $10 p(50 \%)$ & $9 p(40.91 \%)$ & ns \\
\hline Medium (7-9) & $6 \mathrm{p} \mathrm{(30 \% )}$ & $8 p(36.37 \%)$ & ns \\
\hline \begin{tabular}{l|l} 
& High (10-12) \\
\end{tabular} & $4 p(20 \%)$ & $5 p(22.73 \%)$ & ns \\
\hline ns-insignificant & & & \\
\hline
\end{tabular}


for surgeries were it was used the Pringle maneuver. The average clamping time of the renal pedicle with Satinsky clamp was $14.7 \pm 2.3$ minutes.

Average blood loss for all surgeries was $220 \pm 30 \mathrm{ml}$, with $195 \pm 25 \mathrm{ml}$ for surgeries were it was used the Satinsky clamp and $227 \pm 25 \mathrm{ml}$ for surgeries were it was used the Pringle maneuver. The suture of collecting system was made at $8 \mathrm{p}(40 \%)$ in the Satinsky group and at $10 \mathrm{p}$ $(44.45 \%)$ in the Pringle group. The intraoperative characteristic are presented in Table 2.

Renal function, evaluated trought serum creatinine, was better in Pringle maneuver group than the Satinsky clamp group. The results about creatinine value before surgery, after surgery at 1 month and 6 month are shown in Table 3 and 4 .

\section{DISCUSSION}

Renal tumor size was between $1.5 \mathrm{~cm}$ and $7.3 \mathrm{~cm}$ and had no influence on the outcome of the surgery. In order to have an objectiv comparison of the renal tumors between the two groups we utilized P.A.D.U.A. score and R.E.N.A.L. nephrometry scoring system.

Some authors had demonstrate that open partial nephrectomy provides good oncological control for tumors of $7 \mathrm{~cm}$ or less, with indications of tumor in an anatomical or functional solitary kidney $(3,5)$.

All patients in the study benefit from open partial nephrectomy with warm ischemia using Pringle maneuver and Satinsky clamp. The average clamping time of the renal pedicle with Satinsky clamp was $14.7 \pm 2.3$ minutes.

Renal function, evaluated through serum creatinine, was better in Pringle maneuver group (0.64-1.13 $\mathrm{mg} 7 / \mathrm{dl})$ than the Satinsky clamp group (0.84-1.19 mg/dl).

Pringle maneuver has been used for renal tumors on unique surgical or congenital kidneys, multifocal tumors or mediorenal tumors near renal sinus, because of better intraoperative control and surgeons confort with this approach.

TABLE 2. Intraoperative characteristics

\begin{tabular}{|l|c|c|c|c|}
\hline $\begin{array}{l}\text { Renal pedicle clamping } \\
\text { Parameters }\end{array}$ & Satinsky clamp & Pringle maneuver & Overall & $p$ \\
\hline Patients number & $20 p$ & $22 p$ & $42 p$ & $n s$ \\
\hline Mean age & $56.7 \pm 12.4(32-74)$ & $60.5 \pm 11.6(29-75)$ & $58.7 \pm 14.7(29-75)$ & $\mathrm{ns}$ \\
\hline Average operation time (min) & $94.3 \pm 18.9(75-130)$ & $95.3 \pm 19.8(75-130)$ & $94.8 \pm 19.4(75-130)$ & $\mathrm{ns}$ \\
\hline Average clamping time (min) & $14.7 \pm 2.3(14-20)$ & - & - & $<0.01$ \\
\hline Average blood loss (ml) & $195 \pm 25(190-210)$ & $227 \pm 25(210-250)$ & $220 \pm 30(190-250)$ & $\mathrm{ns}$ \\
\hline Suture of the collecting system & $8(40 \%)$ & $10(45.45 \%)$ & $18(42.85 \%)$ & $\mathrm{ns}$ \\
\hline Hospital stay & $4.8 \pm 2.8(3-9)$ & $5.2 \pm 2.6(4-9)$ & $4.8 \pm 2.6(3-9)$ & $\mathrm{ns}$ \\
\hline
\end{tabular}

TABLE 3. Creatinine value before and after surgery

\begin{tabular}{|l|c|c|c|}
\hline $\begin{array}{l}\text { Renal pedicle clamping } \\
\text { Creatinine value } \mathbf{m g - d l}\end{array}$ & Satinsky clamp & Pringle maneuver & $\mathbf{p}$ \\
\hline Before surgery & $0.64-2.1$ & $0.84-3.2$ & $\mathrm{~ns}$ \\
\hline $\mathbf{1}$ month after surgery & $0.92-1.51$ & $0.91-1.28$ & $\mathrm{~ns}$ \\
\hline $\mathbf{6}$ month after surgery & $0.84-1.19$ & $0.64-1.13$ & $\mathrm{~ns}$ \\
\hline
\end{tabular}

TABEL 4. Creatinine value befor and after surgery

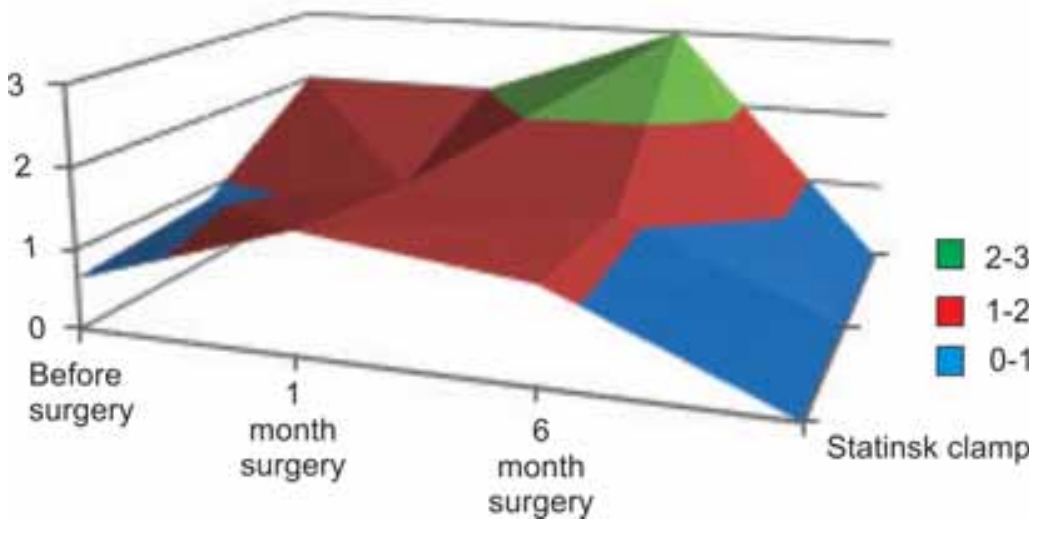


In the studied group there was no tumor recurrence, the follow up period was between 1 and 5 years.

Some studies demonstrated that intermittent renal arterial occlusion should be avoided, since it potentiates arterial vasospasm and therefore renal damage (6), in our study the creatinine value is better in the group that we used the Pringle maneuver.

Matthew F et al. reported that partial nephrectomy with and without clamping in solitary kidneys suggests that renovascular clamping is the only statistically significant determinant of postoperative renal dysfunction. He shows that ischemia is associated with a risk of acute renal failure, advanced CKD, and renal replacement therapy (7).

Some studies have suggested that a solitary kidney is more resistant to ischemic damage compared with both kidneys. In fact, studies on solitary kidneys may overestimate the maximal renal tolerance to ischemia of patients with a normal contralateral renal unit $(8,9)$.

A 2013 paper provided the first detailed analysis of structural and functional responses of the human kidney to controlled clamp ischemia in 40 patients undergoing oper partial nephrectomy with warm ischemia times $>30 \mathrm{~min}$. in $82.5 \%$ of the cases (10). The authors showed resistance to ischemia time of human kidneys, with little or no acute renal failure in the early postoperative period. Progressive levels of ischemia up to $61 \mathrm{~min}$. had no effect on the injury produced.

The temporal trend of loss of RF has been diffusely investigated, especially in the setting of bilateral kidneys. RF appears to decrease immediately after $\mathrm{PN}$ and to partially recover to reach a new steady state, generally within 3 weeks to 3 months after PN (11).

Manual or instrumental compression of the renal parenchyma to control the intraoperative hemorrhage has the advantages of avoiding ischemia of the normal parenchyma, and allowing the surgen to operate in an almost bloodless field. Animal studies have shown that the use of renal parenchyma compression may be more deleterious than simple arterial occlusion (12, 13).

\section{CONCLUSION}

Pringle maneuver is a safe and effective method with low intra and postoperative complications during open partial nephrectomy, having a better control of the renal pedicle with reduced ischemia.

Is a method of choice in renal tumour tumor pathology on a single congenital or surgical kidney, mediorenal tumors or near the sinus and multifocal tumors.

Acknowledgements: The authors declare no conflict of interests and no sponsorship was provided by the manufacturer of the catheters involved in this study. All authors have read and approved this publication and had equal scientific contribution in publishing this material.

\section{REFERENCES}

1. A.C. Novick and S.C. Campbell, Tumores renales, in Campblee Urology, P.C. Walsh, A.B. Retik, E.D. Vaughan, A.J. Wein, Eds. pp2911-2979, 2005.

2. B. Shuch, J.S. Lam, A.S. Belldegrun, Open partial nephrectomy for the treatment of renal cell carcinoma, Current Urology Reports, vol.7, no.1, pp.31-38,2006.

3. A.M. Nieder, S. S. Taneja, The role of partial nephrectomy for renal cell carcinoma in contemporary practice, Urologic Clinics of North America, vol 30, no.3, pp.529-542, 2003.

4. Daniel Canter et al. Utility of the R.E.N.A.L.-Nephrometry Scoring System in Objectifying Treatment Decision-Making of the Enhancing Renal Mass. Urology. 2011 November ; 78(5): 1089-1094.

5. Darren Desantis et al. The association between renal tumour scoring system components and complications of partial nephrectomy. Can Urol Assoc J 2015; 9(1-2):39-45.

6. 30 D.H. Wilson, B.B. Barton, W.L. Parry, L.B. Hinshaw Effects of intermittent versus continuous renal arterial occlusion on hemodynamics and function of the kidney, Invest Urol, 8 (1971), pp. 507-515.

7. Matthew F. Wszolek, Patrick A. Kenney, John A. Libertino Nonclamping partial nephrectomy: towards improved nephron sparing, Nature Reviews Urology 8, 523527 (September 2011) | doi:10.1038/ nrurol.2011.103.

8. A. Askari, A.C. Novick, B.H. Stewart, R.A. Straffon, Surgical treatment of renovascular disease in the solitary kidney: results in 43 cases J Urol, 127 (1982), pp. 20-22.

9. P. Jablonski, B. Howden, D. Rae et al. The influence of the contralateral kidney upon recovery from unilateral warm renal ischemia Pathology, 17 (1985), pp. 623-627.
10. S.C. Campbell, A.C. Novick, A. Belldegrun et al. Guideline for management of the clinical T1 renal mass $J$ Urol, 182 (2009), pp. 1271-1279.

11. F. Porpiglia, C. Fiori, R. Bertolo et al. The effects of warm ischemia time on renal function after laparoscopic partial nephrectomy in patients with normal contralateral kidney, World J Urol, 30 (2012), pp. 257-263.

12. Neely W.A., Turnet M.D. The effect of arterial, venous, and arteriovenous occlusion on renal blood flow. Surgery, Gynecology and Obstetrics. 1959:108(6):669-672.

13. Schirmer H.K. The effect of intermittent and prolonged renal artery occlusion upon respiration and anaerobic glycolysis of dog kidney. The Journal of Urology: 1965:94(5):511-513. 